\title{
Electron-phonon interaction and coupled phonon-plasmon modes
}

\author{
L. A. Falkovsky \\ Landau Institute for Theoretical Physics, 117337 Moscow, Russia
}

November 4, 2018

\begin{abstract}
The theory of Raman scattering by the electron-phonon coupled system in metals and heavily doped semiconductors is developed taking into account the Coulomb screening and the electron-phonon deformation interaction. The Boltzmann equation for carriers is applied. Phonon frequencies and optic coupling constants are renormalized due to interactions with carriers. The $k$-dependent semiclassical dielectric function is involved instead of the LindhardMermin expression. The results of calculations are presented for various values of carrier concentration and electron-phonon coupling constant.

PACS number(s): 63.20.Dj, 63.20.Kr, 71.38-k, 72.30.+q, 78.30.-j
\end{abstract}

\section{Introduction}

Recently there has been considerable interest in the effect of electron-phonon interactions on the optical-phonon dispersion. This interest is stimulated by contradictions between different approaches to the electron-phonon interaction. The strong phonon renormalizations were obtained first by Migdal [1 (see also 2]) within a consistent many-body approach based on the Frölich Hamiltonian. The extremely large dispersion of optical phonons was predicted in work [3] using also the Frölich model. These results contradict to the Born-Oppenheimer (adiabatic) concept 4 according to which the phonon renormalizations should be small in terms of the nonadiabatic parameter $\sqrt{m / M}$, where $m$ and $M$ are the electron and ion masses, respectively (see, also [5]). Theoretical investigations [6] of the sound velocity and acoustic attenuation in metals confirm the adiabatic concept. In a recent paper, Reizer [7] emphasized the importance of screening which should be taken into account. To our knowledge, the Coulomb screening effect on the LO phonons was firstly studied in the work [8]. In our work [9], using the Boltzmann equation we have found 
that the electron-phonon interaction results more essentially in the optical-phonon damping than in the dispersion law. In any case, the Frölich model has the evident shortcomings.

From the experimental point of view, the best opportunity for the investigation of interactions between electrons and optical phonons is provided by coupled phononplasmon modes in doped semiconductors (see, e.g. [10]). Two such modes $L^{ \pm}$have been observed in Raman experiments for many semiconductors. On the early step, the Raman results were compared with the theory [11] based on a Drude model (see, e.g. [12]), but more recently the Lindhard-Mermin expression for the dielectric function has been used [13.

The Lindhard-Mermin expression [14] represents a sophisticated generalization of the Lindhard function with the help of the electron relaxation time. The Lindhard approach is very useful while the momentum transfer $k$ in the Raman scattering is compared with the Fermi momentum $p_{F}$. The most essential influence of carriers should be expected for $k v_{F} \sim \omega$, where $v_{F}$ is the Fermi velocity and $\omega$ is the phonon frequency. For solids with metalic conductivity, the Fermi velocity could be estimated using an argument of stability under the Coulomb interaction $e^{2} / \pi \hbar v_{F} \leq 1$. This condition gives $v_{F} \sim 0.7 \times 10^{8} \mathrm{~cm} / \mathrm{s}$. For the typical value of the optical phonon frequency $\omega=500 \mathrm{~cm}^{-1}$, the interesting values $k \leq \omega / v_{F} \simeq 10^{6} \mathrm{~cm}^{-1}$. Therefore, the condition $k<p_{F}$ is satisfied for the carrier concentration larger than $3 \times 10^{17} \mathrm{~cm}^{-3}$. In experiments, the heavily doped semiconductors with large carrier concentration are used, in order to obtain a visible effect of carriers. In such a situation, the condition $k \ll p_{F}$ is fulfilled, and we can apply the Boltzmann equation in calculations of electronic susceptibility, as well as for the evaluation of the Raman cross section. The method of the Boltzmann equation is valid for the anisotropic electron plasma in solids at arbitrary temperatures. In the present paper, we obtain the Raman efficiency applying the Boltzmann equation for generate carriers in heavily doped semiconductors when temperature is lower than the Fermi energy, $T \ll \varepsilon_{F}$.

\section{Effective Hamiltonian and light scattering}

For the electron-phonon system in solids, we use the operator of particle numbers $\hat{n}$, the phonon displacements $\hat{b}_{j}$, and the macroscopic electric field $E$, accompanying vibrations in polar semiconductors and acting on charges of electrons and ions. The effective Hamiltonian describing the inelastic light scattering in solids can be written in the semiclassical Wigner representation

$$
\mathcal{H}=\frac{e^{2}}{m c^{2}} \int d^{3} r \mathcal{N}(\mathbf{r}, t) U(\mathbf{r}, t)
$$

where

$$
\mathcal{N}(\mathbf{r}, t)=\gamma \hat{n}(\mathbf{r}, t)+g_{j} \hat{b}_{j}(\mathbf{r}, t)+g_{E} E(\mathbf{r}, t)
$$


is a linear form of variables $\hat{n}, \hat{b}_{j}$, and $E$. The subscript $j$ denotes the various phonon modes: longitudinal (LO) or transverse (TO). More precisely, the subscript $j$ numerates the different phonon representations which can be degenerate. The transformation properties of the coupling constants $g_{j}$ are determined by this representation. The notation $U(\mathbf{r}, t)$ is introduced for a product of the vector-potentials of incident and scattered photons:

$$
A^{(i)}(\mathbf{r}, t) A^{(s)}(\mathbf{r}, t)=U(\mathbf{r}, t)=\exp [i(\mathbf{k} \cdot \mathbf{r}-\omega t)] U(\mathbf{k}, \omega)
$$

where the momentum and frequency transfers $\mathbf{k}=\mathbf{k}^{(i)}-\mathbf{k}^{(s)}, \omega=\omega^{(i)}-\omega^{(s)}$. The polarization vectors of $\hat{\mathbf{b}}_{j}(\mathbf{r}, t), \mathbf{E}(\mathbf{r}, t), \mathbf{A}^{(i)}(\mathbf{r}, t)$, and $\mathbf{A}^{(s)}(\mathbf{r}, t)$ are included in the coupling constants.

The first term on the right-hand side of Eq. (2) descibes the light scattering by electron-hole pairs with a vertex

$$
\gamma(\mathbf{p})=e_{\alpha}^{(i)} e_{\beta}^{(s)}\left[\delta_{\alpha \beta}+\frac{1}{m} \sum_{n}\left(\frac{p_{f n}^{\beta} p_{n f}^{\alpha}}{\epsilon_{f}(\mathbf{p})-\epsilon_{n}(\mathbf{p})+\omega^{(i)}}+\frac{p_{f n}^{\beta} p_{n f}^{\alpha}}{\epsilon_{f}(\mathbf{p})-\epsilon_{n}(\mathbf{p})-\omega^{(s)}}\right)\right]
$$

where the resonant term is included; $e_{\alpha}^{(i)}$ and $e_{\beta}^{(s)}$ are the polarizatin vectors of incident $\mathbf{A}^{(i)}(\mathbf{r}, t)$ and scattered $\mathbf{A}^{(s)}(\mathbf{r}, t)$ photons. The quantum-mechanical and statistical average of the first term in Eq. (2)

$$
\langle\langle\gamma \hat{n}(\mathbf{r}, t)\rangle\rangle=\int \frac{2 d^{3} p}{(2 \pi)^{3}} \gamma(\mathbf{p}) f_{p}(\mathbf{r}, t)
$$

can be expressed in terms of the electron distribution function $f_{p}(\mathbf{r}, t)$. The constants $g_{j}$ and $g_{E}$ are the deformation-optic and electro-optic couplings with the phonon displacements and the macroscopic electric field, correspondingly. The estimation gives $g_{j} \sim / a^{4}, g_{E} \sim 1 / e a$, and $\gamma(\mathbf{p}) \sim m^{*} / m$, where $a$ is the lattice parameter and $m^{*}$ is the effective mass.

The variable $U(\mathbf{r}, t)$ can be considered as an external force. Then one introduces the generalized susceptibility $\chi(\mathbf{k}, \omega)$ as the linear response to this force:

$$
\langle\langle\mathcal{N}(\mathbf{k}, \omega)\rangle\rangle=-\chi(\mathbf{k}, \omega) U(\mathbf{k}, \omega) .
$$

According to the fluctuation-dissipation theorem the function

$$
K(\mathbf{k}, \omega)=\frac{2}{1-\exp (-\omega / T)} \operatorname{Im} \chi(\mathbf{k}, \omega)
$$

is the Fourier component of the correlation function

$$
K\left(\mathbf{r}, t ; \mathbf{r}^{\prime}, t^{\prime}\right)=\left\langle\left\langle\mathcal{N}^{\dagger}(\mathbf{r}, t) \mathcal{N}\left(\mathbf{r}^{\prime}, t^{\prime}\right)\right\rangle\right\rangle
$$


which depends only on the differences $\mathbf{r}-\mathbf{r}^{\prime}$ and $t-t^{\prime}$. The Raman cross section is given by

$$
\frac{d \sigma}{d \omega^{(s)} d \Omega^{(s)}}=\frac{k_{z}^{(s)} \omega^{(s)}}{\pi c}\left(\frac{2 e^{2}}{c \hbar m \omega^{(i)}}\right)^{2} K(\mathbf{k}, \omega)|U(\mathbf{k}, \omega)|^{2},
$$

where $k_{z}^{(s)}$ is the normal to the sample surface component of the scattered wave vector in vacuum.

One note should be made. Of course, any sample has the surface. The surface effects in the Raman scattering was considered in our work [15] and they are omitted in the derivation of $\mathrm{Eg}$. (6). Furthermore, the incident and scattered fields do not penetrate into the bulk due to the skin effect. For the optical range of the incident light, we have the normal skin-effect conditions. Then we integrate in Eq. (6) the distribution $|U(\mathbf{k}, \omega)|^{2}$ over the normal component $k_{z}$. As shown in the paper [15], the integration of $|U(\mathbf{k}, \omega)|^{2}$ gives a factor $1 / \zeta_{2}$, where $\zeta_{2}$ is expressed in terms of the wavevector components inside semiconductor: $\zeta_{2}=\operatorname{Im}\left(k_{z}^{(i)}+k_{z}^{(s)}\right)$. The obtained Raman cross section (6) is dimensionless. It represents a ratio of the inelastic scattered light energy to the incident energy.

\section{Boltzmann equation for carriers}

The problem of the evaluation of the Raman cross section consists in the calculation of the generalized susceptibility (44). We apply the Boltzmann equation for the electron distribution function:

$$
\frac{\partial f_{p}(\mathbf{r}, t)}{\partial t}+\mathbf{v} \frac{\partial f_{p}(\mathbf{r}, t)}{\partial \mathbf{r}}+\dot{\mathbf{p}} \frac{\partial f_{p}(\mathbf{r}, t)}{\partial \mathbf{p}}=-\frac{1}{\tau}\left[f_{p}(\mathbf{r}, t)-\left\langle f_{p}(\mathbf{r}, t)\right\rangle\right] .
$$

The angular brackets denote the average over the Fermi surface

$$
\langle\ldots\rangle=\frac{1}{\nu_{0}} \int(\ldots) \frac{2 d S_{F}}{v(2 \pi)^{3}}
$$

where the integral is performed in the momentum space over the Fermi surface and $\nu_{0}$ is the density of electron states, defined by the condition $\langle 1\rangle=1$. We use the $\tau$-approximation which is correct for the electron scattering by defects in metals and heavily doped semiconductors. The collision integral in the form (17) conserves the number of electrons in collisions. Therefore, the charge density satisfies the equation of continuity. This ensures the correct $\omega$-dependance of the dielectric function at low frequencies.

According to Eqs. (11), (2) and (3) instead of the unperturbed electron spectrum $\varepsilon_{0}(\mathbf{p})$, we introduce the local electron spectrum in the presence of the external force $U(\mathbf{r}, t)$ as follows:

$$
\varepsilon(\mathbf{p}, \mathbf{r}, t)=\varepsilon_{0}(\mathbf{p})+\gamma(\mathbf{p}) U(\mathbf{r}, t)+\zeta_{j}(\mathbf{p}) b_{j}(\mathbf{r}, t),
$$


where the last term represents the electron-optical-phonon deformation potential and $b_{j}(\mathbf{r}, t)=\left\langle\left\langle\hat{b}_{j}(\mathbf{r}, t)\right\rangle\right\rangle$. We use this form of the el-ph interaction instead of the Frölich poirization type $\zeta(\mathbf{p}) \operatorname{div} \mathbf{b}(\mathbf{r}, t)$, because it is larger by the parameter $1 / k a$ for the optical phonons.

Let us linearize Eq. (7), looking for its solution in the form

$$
f_{p}(\mathbf{r}, t)=f_{0}[\varepsilon(\mathbf{p}, \mathbf{r}, t)-\mu]-\frac{d f_{0}}{d \varepsilon} \delta f_{p}(\mathbf{r}, t),
$$

where $f_{0}[\varepsilon(\mathbf{p}, \mathbf{r}, t)-\mu]$ is the Fermi-Dirac local distribution function. It is important that the collision term in the Boltzmann equation is canceled by the local-equilibrium term of Eq. (8).

We set the number-conservation condition on the chemical potential:

$$
\int \frac{d^{3} p}{(2 \pi)^{3}} f_{0}[\varepsilon(\mathbf{p}, \mathbf{r}, t)-\mu]=\int \frac{d^{3} p}{(2 \pi)^{3}} f_{0}\left(\varepsilon_{0}-\mu_{0}\right)
$$

We obtain

$$
\mu=\mu_{0}+\langle\gamma(\mathbf{p})\rangle U(\mathbf{r}, t)+\left\langle\zeta_{j}(\mathbf{p})\right\rangle b_{j}(\mathbf{r}, t) .
$$

The condition implies the renormalization of vertices

$$
\gamma(\mathbf{p}) \rightarrow \gamma(\mathbf{p})-\langle\gamma(\mathbf{p})\rangle, \quad \zeta_{j}(\mathbf{p}) \rightarrow \zeta_{j}(\mathbf{p})-\left\langle\zeta_{j}(\mathbf{p})\right\rangle
$$

and this substitution should be made in that follows.

The linearized Boltzmann equation in the Fourier components has the form

$$
-i(\omega-\mathbf{k} \cdot \mathbf{v}+i / \tau) \delta f_{p}(\mathbf{k}, \omega)=\psi_{p}(\mathbf{k}, \omega)+\left\langle\delta f_{p}(\mathbf{k}, \omega)\right\rangle / \tau,
$$

where

$$
\psi_{p}(\mathbf{k}, \omega)=e \mathbf{v} \cdot \mathbf{E}(\mathbf{k}, \omega)-i \omega\left[\gamma(\mathbf{p}) U(\mathbf{k}, \omega)+\zeta_{j}(\mathbf{p}) b_{j}(\mathbf{k}, \omega)\right] .
$$

The solution to the equation is esily obtained:

$$
\delta f_{p}(\mathbf{k}, \omega)=i\left[\psi_{p}(\mathbf{k}, \omega)+\left\langle\delta f_{p}(\mathbf{k}, \omega)\right\rangle / \tau\right] / \Delta_{p},
$$

where we denote $\Delta_{p}=\omega-\mathbf{k} \cdot \mathbf{v}+i / \tau$. Now we get

$$
\left\langle\delta f_{p}(\mathbf{k}, \omega)\right\rangle=i\left\langle\psi_{p}(\mathbf{k}, \omega) / \Delta_{p}\right\rangle /\left(1-i\left\langle\tau^{-1} / \Delta_{p}\right\rangle\right) .
$$

Notice, that in accordance with the adiabatic concept, no additional contribution comes from the local equilibrium distribution function $f_{0}[\varepsilon(\mathbf{p}, \mathbf{r}, t)]$ in Eq. (8) $)$. 


\section{Equation of motion for phonons interacting with carriers}

In the long-wave approximation ( $k \ll 1 / a, a$ being the lattice parameter), we write the equation of motion for the phonon displacement field

$$
\left(\omega_{k}^{2}-\omega^{2}\right) b_{j}(\mathbf{k}, \omega)=\frac{Z}{M^{\prime}} E_{j}(\mathbf{k}, \omega)-\frac{g_{j} U(\mathbf{k}, \omega)}{M^{\prime} N}-\frac{1}{M^{\prime} N} \int \frac{2 d S_{F}}{v(2 \pi)^{3}} \zeta_{j}(\mathbf{p}) \delta f_{p}(\mathbf{k}, \omega)
$$

where $N$ is the number of unit cells in $1 \mathrm{~cm}^{3}, M^{\prime}$ is the reduced mass of the unit cell, and $Z$ is the effective ionic charge. The nonperturbed phonon frequency $\omega_{k}$ should be considered in the absence of the electric field and without any electronphonon interactions. In the long-wave limit, we can expand it as $\omega_{k}^{2}=\omega_{0}^{2} \pm s^{2} k^{2}$ with the value of the dispersion parameter $s$ on the order of the typical sound velocity in solids. Notice that the optical phonons always have the so-called natural width $\Gamma^{\text {nat }} \sim \omega_{0} \sqrt{m / M}$. The natural width results from decay processes into two or more acoustic and optical phonons. In the final expressions, we will substitute $\omega_{k}^{2}-\omega^{2} \rightarrow$ $\omega_{k}^{2}-i \omega \Gamma^{\text {nat }}-\omega^{2}$.

The equation (12) is applied to both the longitudinal and transverse phonons. It is seen from the Maxwell equations, that the electric field is longitudinal $\mathbf{E} \| \mathbf{k}$ in the optical region $k \gg \omega / c$. If the excited phonons propagate in the symmetrical direction, the TO and LO phonons are separated. Therefore, the electric field stands only in Eq. (12) for the LO phonon. Beside of that, the coupling $\zeta_{j}(\mathbf{p})$ depends on the phonon representation $j$.

Using the solution (10), we rewrite Eq. (12):

$$
\left(\tilde{\omega}_{j}^{2}-\omega^{2}\right) b_{j}(\mathbf{k}, \omega)-\frac{\tilde{Z}}{M^{\prime}} E_{j}(\mathbf{k}, \omega)=-\frac{\tilde{g}_{j} U(\mathbf{k}, \omega)}{M^{\prime} N},
$$

where the phonon frequency

$$
\tilde{\omega}_{j}^{2}=\omega_{k}^{2}+\frac{\omega \nu_{0}}{M^{\prime} N}\left(\left\langle\frac{\zeta_{j}^{2}(\mathbf{p})}{\Delta_{p}}\right\rangle+\frac{i\left\langle\zeta_{j}(\mathbf{p}) / \Delta_{p}\right\rangle^{2}}{\tau-\left\langle i / \Delta_{p}\right\rangle}\right),
$$

the effective ionic charge

$$
\tilde{Z}=Z-\frac{i e \nu_{0}}{N}\left(\left\langle\frac{v_{z} \zeta_{j}(\mathbf{p})}{\Delta_{p}}\right\rangle+\frac{i\left\langle v_{z} / \Delta_{p}\right\rangle\left\langle\zeta_{j}(\mathbf{p}) / \Delta_{p}\right\rangle}{\tau-\left\langle i / \Delta_{p}\right\rangle}\right),
$$

and the deformation-optic coupling

$$
\tilde{g}_{j}=g_{j}+\omega \nu_{0}\left(\left\langle\frac{\zeta_{j}(\mathbf{p}) \gamma(\mathbf{p})}{\Delta_{p}}\right\rangle+\frac{i\left\langle\zeta_{j}(\mathbf{p}) / \Delta_{p}\right\rangle\left\langle\gamma(\mathbf{p}) / \Delta_{p}\right\rangle}{\tau-\left\langle i / \Delta_{p}\right\rangle}\right)
$$

are renormalized due to the electron-phonon interaction $\zeta_{j}(\mathbf{p})$. 


\section{$5 \quad$ Poisson equation for the macroscopic field}

Let us consider the longitudinal electric induction $D$ that accompanies the lattice vibrations. There are several contributions in the field : (1) the polarization $\alpha E(\mathbf{r}, t)$ of the filled electron bands, (2) the lattice polarization $N Z b_{\mathrm{LO}}(\mathbf{r}, t),(3)$ the contribution of free carrier density $\rho=-\operatorname{div} \mathbf{P}_{e}$, and (4) the term $P=-\partial \mathcal{H} / \partial E=-g_{E} U$

explicitly results from the Hamiltonian (11), (2). Collecting all these terms into the Poisson equation, $\operatorname{div} \mathbf{D}=0$, we find

$$
\varepsilon_{\infty} E(\mathbf{k}, \omega)+4 \pi N Z b_{\mathrm{LO}}(\mathbf{k}, \omega)+\frac{4 \pi i e}{k} \int \frac{2 d^{3} p}{(2 \pi)^{3}} \delta f_{p}(\mathbf{k}, \omega)-4 \pi g_{E} U(\mathbf{k}, \omega)=0,
$$

where the high-frequency permittivity $\varepsilon_{\infty}=1+4 \pi \alpha$. Using the solution of the Boltzmann equation we rewrite the Poisson equation in the form

$$
\varepsilon_{e}(\mathbf{k}, \omega) E(\mathbf{k}, \omega)+4 \pi N \bar{Z} b_{\mathrm{LO}}(\mathbf{k}, \omega)=4 \pi \tilde{g}_{E} U(\mathbf{k}, \omega),
$$

where the electronic dielectric function

$$
\varepsilon_{e}(\mathbf{k}, \omega)=\varepsilon_{\infty}+\varepsilon_{\infty} \frac{k_{0}^{2}}{k^{2}}\left[1-\frac{\left\langle\omega / \Delta_{p}(k)\right\rangle}{1-\left\langle i / \Delta_{p}(k)\right\rangle / \tau}\right]
$$

with the Thomas-Fermi parameter $k_{0}^{2}=4 \pi e^{2} \nu_{0} / \varepsilon_{\infty}$.

Due to the electron-phonon interactions $\zeta_{\mathrm{LO}}(\mathbf{p})$, the ionic charge obtains an additional term

$$
\bar{Z}=Z+\frac{i e \nu_{0}}{N}\left(\left\langle\frac{v_{z} \zeta_{\mathrm{LO}}(\mathbf{p})}{\Delta_{p}}\right\rangle+\frac{i\left\langle v_{z} / \Delta_{p}\right\rangle\left\langle\zeta_{\mathrm{LO}}(\mathbf{p}) / \Delta_{p}\right\rangle}{\tau-\left\langle i / \Delta_{p}\right\rangle}\right)
$$

of the opposite sign in the comparison with that in Eq. (15). The electro-optic coupling in Eq. (17) also changes, but because of the light scattering by carriers $\gamma(\mathbf{p})$ :

$$
\tilde{g}_{E}=g_{E}-i e \nu_{0}\left(\left\langle\frac{v_{z} \gamma(\mathbf{p})}{\Delta_{p}}\right\rangle+\frac{i\left\langle v_{z} / \Delta_{p}\right\rangle\left\langle\gamma(\mathbf{p}) / \Delta_{p}\right\rangle}{\tau-\left\langle i / \Delta_{p}\right\rangle}\right) .
$$

\section{Raman scattering by electron-hole pairs, phonons, and coupled modes}

Now we are in a position to calculate the susceptibility (44). Using Eqs. (3), (10), (11), (16), and (21), we get

$$
\langle\langle\mathcal{N}(\mathbf{k}, \omega)\rangle\rangle=-\chi_{e}(\mathbf{k}, \omega) U(\mathbf{k}, \omega)+\tilde{g}_{j} b_{j}(\mathbf{k}, \omega)+\bar{g}_{E} E(\mathbf{k}, \omega),
$$

where

$$
\chi_{e}(\mathbf{k}, \omega)=-\omega \nu_{0}\left(\left\langle\frac{\gamma^{2}(\mathbf{p})}{\Delta_{p}}\right\rangle+\frac{i\left\langle\gamma(\mathbf{p}) / \Delta_{p}\right\rangle^{2}}{\tau-\left\langle i / \Delta_{p}\right\rangle}\right)
$$


gives the light scattering with excitation of electron-hole pairs. Notice, that here the renormalized coupling $\bar{g}_{E}$ differs from $\tilde{g}_{E}(21)$ in the sign of the second term:

$$
\bar{g}_{E}=g_{E}+i e \nu_{0}\left(\left\langle\frac{v_{z} \gamma(\mathbf{p})}{\Delta_{p}}\right\rangle+\frac{i\left\langle v_{z} / \Delta_{p}\right\rangle\left\langle\gamma(\mathbf{p}) / \Delta_{p}\right\rangle}{\tau-\left\langle i / \Delta_{p}\right\rangle}\right) .
$$

In order to find $E(\mathbf{k}, \omega)$ and $b_{j}(\mathbf{k}, \omega)$, we have to solve the system of the algebraic equations (13) and (18). Then, using Eq. (22) we obtain the generalized susceptibility

$$
\chi(\mathbf{k}, \omega)=\chi_{e}(\mathbf{k}, \omega)+\frac{\tilde{g}_{j}^{2} \varepsilon_{e}(\mathbf{k}, \omega) / N M^{\prime}-4 \pi \tilde{g}_{E} \bar{g}_{E}\left(\tilde{\omega}_{j}^{2}-\omega^{2}\right)-4 \pi \tilde{g}_{j}\left(\tilde{g}_{E} \widetilde{Z}+\bar{g}_{E} \bar{Z}\right) / M^{\prime}}{\left(\tilde{\omega}_{j}^{2}-\omega^{2}\right) \varepsilon_{e}(\mathbf{k}, \omega)+4 \pi N \widetilde{Z} \bar{Z} / M^{\prime}} .
$$

The expression (23) is our main result. Here the poles of the second term give the spectrum of collective excitations of the electron-phonon system. We discuss Eq. (23) in various limiting cases.

\section{The electronic scattering}

We obtain the Raman electronic scattering from Eq. (23), if we set $\tilde{g}_{j}=g_{E}=\widetilde{Z}=$ $\bar{Z}=0$. We get

$$
\chi(\mathbf{k}, \omega)=\chi_{e}(\mathbf{k}, \omega)+\frac{4 \pi \tilde{g}_{E}^{2}}{\varepsilon_{e}(\mathbf{k}, \omega)},
$$

where $\tilde{g}_{E}$ is given in Eq. (21) with $g_{E}=0$.

For the isotropic Fermi surface, we calculate the dielectric function Eq. (19) performing the integration:

$$
\left\langle 1 / \Delta_{p}(k)\right\rangle=\frac{1}{2 k v_{F}} \ln \frac{1+\kappa}{1-\kappa}, \quad \kappa=k v_{F} /\left(\omega+i \tau^{-1}\right),
$$

where one should take the branch of $\ln x$, which is real for positive real values of $x$.

For the anisotropic Fermi surface, the calculations can be done in limiting cases. For $|\kappa| \gg 1$, we use the expansion for the electronic dielectric function (19):

$$
\varepsilon_{e}(\mathbf{k}, \omega)=\varepsilon_{\infty}\left\{1+\left(k_{0} / k\right)^{2}\left[1+i\left(\pi \nu_{0} \omega / k\right)\langle\delta(\mu) / v\rangle\right]\right\},
$$

where $\mu=\mathbf{v} \cdot \mathbf{k} / v k$ and $\delta(x)$ is the Dirac delta-function. In this case, the Raman efficiency has a "tail" due to the Landau damping:

$$
\operatorname{Im} \chi(\mathbf{k}, \omega)=\left(\pi \nu_{0} \omega / k\right)\left\langle\gamma^{2}(\mathbf{p}) \delta(\mu) / v\right\rangle .
$$

We see, that the Raman cross section vanishes for the isotropic vertex $\gamma(\mathbf{p})$ because of Eq. (9). This is a result of the Coulomb screening. It was obtained first in the work [16] for the Raman scattering in semiconductors (see, [17]). 
In the opposite case $|\kappa| \ll 1$, the first term in Eq. (24) gives a result

$$
\operatorname{Im} \chi_{e}(\mathbf{k}, \omega)=\nu_{0}\left\langle\gamma^{2}(\mathbf{p})\right\rangle \frac{\omega \tau}{(\omega \tau)^{2}+1}
$$

which was found first in the work 18, with a help of the Green's function technique. The second term in Eq. (24) reveals a plasmon pole at small values of $k$. The $k$-expansion of the dielectric function reads

$$
\varepsilon_{e}(k, \omega)=\varepsilon_{\infty}\left(1-\frac{\omega_{p e}^{2}+k^{2} w}{\omega\left(\omega+i \tau^{-1}\right)}\right),
$$

where the $k$-independent term represents the Drude conductivity and the electron plasma frequency is given by the integral over the Fermi surface $\omega_{p e}^{2}=k_{0}^{2}\left\langle v_{z}^{2}\right\rangle$. The complex coefficient $w=k_{0}^{2}\left(\left\langle v_{z}^{4}\right\rangle+i\left\langle v_{z}^{2}\right\rangle^{2} / \omega \tau\right) /\left(\omega+i \tau^{-1}\right)^{2}$. For the isotropic electron spectrum and in collisionless regime, $\tau^{-1}=0$, the coefficient $w=(3 / 5) \omega_{p e}^{2} v_{F}^{2}$.

The $k$-expansion of $\tilde{g}_{E}$ gives

$$
\tilde{g}_{E}=-i e \nu_{0} k\left\langle\gamma(\mathbf{p}) v_{z}^{2}\right\rangle /\left(\omega+i \tau^{-1}\right)^{2},
$$

because $g_{E}=0$ and the zero-order term in the $k$-expansion vanishes due to the time invariance $\mathbf{v} \rightarrow-\mathbf{v}$. Then the intensity of the plasmon peak $\sim k^{2}$ in accordance with the known behavior of the dynamical structure factor.

\section{The Raman scattering by TO phonons}

Ihe second term in Eq. (23) gives the TO-phonon scattering, if we set $\widetilde{Z}=\bar{Z}=\tilde{g}_{E}=$ 0 :

$$
\chi(\mathbf{k}, \omega)=\frac{\tilde{g}_{\mathrm{TO}}^{2} / N M^{\prime}}{\tilde{\omega}_{\mathrm{TO}}^{2}-\omega^{2}-i \omega \Gamma^{\text {nat }}},
$$

where $\tilde{\omega}_{\mathrm{TO}}, \tilde{g}_{\mathrm{TO}}$ are defined in Eqs. (14) and (16) with $\zeta_{j}(\mathbf{p})=\zeta_{\mathrm{TO}}(\mathbf{p})$; we add the phonon width $\Gamma^{\text {nat }}$ mentioned above.

Here we should note two points. First, the TO-resonance takes place at the renormalized frequency $\tilde{\omega}_{\text {TO }}$. Taking the real and imaginary parts of the expression (14), we obtain the TO-phonon shift and width due to the deformation interaction $\zeta(\mathbf{p})$ with carriers:

$$
\Delta \omega_{k}=\operatorname{Re}\left(\tilde{\omega}_{\mathrm{TO}}^{2}-\omega_{k}^{2}\right) / 2 \omega_{k}, \quad \Gamma=\Gamma^{\mathrm{nat}}-\operatorname{Im} \tilde{\omega}_{\mathrm{TO}}^{2} / \omega_{k} .
$$

Second, because of the interaction with carriers, the coupling $\tilde{g}_{\mathrm{TO}}$ (16) has an imaginary part. Therefore the line-shape of the resonance becomes asymmetric (the Fano resonance):

$$
\operatorname{Im} \chi(\mathbf{k}, \omega)=\frac{1}{N M^{\prime}} \frac{\omega \Gamma g_{\mathrm{TO}}^{2}+\left[\left(\operatorname{Re} \tilde{\omega}_{\mathrm{TO}}\right)^{2}-\omega^{2}\right] \operatorname{Im} \tilde{g}_{\mathrm{TO}}^{2}}{\left[\left(\operatorname{Re} \tilde{\omega}_{\mathrm{TO}}\right)^{2}-\omega^{2}\right]^{2}+(\omega \Gamma)^{2}}
$$


The line-shape asymmetry depends on the sign of $\operatorname{Re} g_{\mathrm{TO}}$. For instance, if $\operatorname{Re} g_{\mathrm{TO}}>$ 0 , the high-frequency wing of the resonance line drops more slowly than the lowfrequency one. In the limiting case $\kappa \gg 1$, we expand

$$
\tilde{g}_{\mathrm{TO}}=g_{\mathrm{TO}}+\left(\nu_{0} \omega / k\right)\left\langle\gamma(\mathbf{p}) \zeta_{\mathrm{TO}}(\mathbf{p})(-i \pi+2 \omega / k v) \delta(\mu) / v\right\rangle
$$

and for $\kappa \ll 1$ :

$$
\tilde{g}_{\mathrm{TO}}=g_{\mathrm{TO}}+\frac{\omega \nu_{0}}{\omega+i \tau^{-1}}\left(\left\langle\gamma(\mathbf{p}) \zeta_{\mathrm{TO}}(\mathbf{p})\right\rangle+\frac{k^{2}\left\langle v_{z}^{2} \gamma(\mathbf{p}) \zeta_{\mathrm{TO}}(\mathbf{p})\right\rangle}{\left(\omega+i \tau^{-1}\right)^{2}}\right) .
$$

Notice, that the electron-phonon interaction $\zeta_{\mathrm{TO}}(\mathbf{p})$ and the light scattering $\gamma(\mathbf{p})$ by carriers renormalize jointly the coupling $g_{\mathrm{TO}}$. The frequency renormalization

$\tilde{\omega}_{\text {TO }}^{2}$ [see Eq. (14)] results only from the electron-phonon interaction $\zeta_{\text {TO }}(\mathbf{p})$. The corresponding expressions can be obtained from Eqs. (32) and (33) by substitution $\gamma(\mathbf{p}) \rightarrow \zeta_{\mathrm{TO}}(\mathbf{p})$. We see, that the TO phonons become broader and harder because of the interaction with carriers.

Emphasize, that the phonon renormalizations depend on the carrier density $\nu_{0}$ and the average coupling $\zeta_{j}(\mathbf{p})-\left\langle\zeta_{j}(\mathbf{p})\right\rangle$. They vanish for the isotropic Fermi surface. The maximum value of the relative renormalization has the order of $\lambda a p_{F} m^{*} \omega / m \mid \omega+$ $i \tau^{-1} \mid$ at $k v \sim\left|\omega+i \tau^{-1}\right|$, where $\lambda$ is the dimensionless electron-phonon coupling and $m^{*}$ is effective electron mass.

\section{The Raman scattering by LO-phonon-plasmon coupled modes}

In this case, the carriers interact with each other and the ion vibrations via both the macroscopic electric field $E(\mathbf{r}, t)$ and the deformation potential $\zeta_{\mathrm{LO}}(\mathbf{p})$. In the long-wave limit $k \rightarrow 0$, Eqs. (15), (20), and (21) show no renormalization of the ionic charge, $\widetilde{Z}=\bar{Z}=Z$, and the electro-optic constant, $\tilde{g}_{E}=g_{E}$. The equation (23) takes the form:

$$
\chi(0, \omega)=\chi_{e}(0, \omega)+\frac{\tilde{g}_{\mathrm{LO}}^{2} \varepsilon_{e}(0, \omega) / N M^{\prime}-4 \pi g_{E}^{2}\left(\tilde{\omega}_{0}^{2}-i \omega \Gamma^{\mathrm{nat}}-\omega^{2}\right)-8 \pi g_{E} \tilde{g}_{\mathrm{LO}} Z / M^{\prime}}{\left(\tilde{\omega}_{0}^{2}-i \omega \Gamma^{\text {nat }}-\omega^{2}\right) \varepsilon_{e}(0, \omega)+4 \pi N Z^{2} / M^{\prime}},
$$

where the first term is given in Eq. (28). The deformation potential $\zeta_{\mathrm{LO}}(\mathbf{p})$ renormalizes the phonon frequency $\tilde{\omega}_{0}(14)$, as well as the deformation-optic constant $\tilde{g}_{\mathrm{LO}}$ (16). The corresponding expansion in the limiting cases are similar to Eqs. (32) and (33). All mentioned above about the TO line asymmetry concerns also the LO line.

Because the dielectric function of the electron-ion system reads

$$
\varepsilon(0, \omega)=\varepsilon_{e}(0, \omega)+4 \pi N Z^{2} / M^{\prime}\left(\tilde{\omega}_{0}^{2}-i \omega \Gamma^{\text {nat }}-\omega^{2}\right),
$$

the second term on the right-hand side in Eq. (34) has poles while $\varepsilon(0, \omega)=0$. This condition defines the frequency of coupled phonon-plasmon modes in the long-wave limit. 
In the absence of the electron and phonon collisions $\left(\tau^{-1}=\Gamma^{\text {nat }}=0\right)$, and without the el-ph interaction $(\zeta(\mathbf{p})=0)$, one obtains with the help of Eq. (29) the biquadratic equation. It gives the frequencies of the coupled phonon-plasmon modes at $k=0$ :

$$
\omega_{ \pm}^{2}=\frac{1}{2}\left(\omega_{p e}^{2}+\omega_{\mathrm{LO}}^{2}\right) \pm \frac{1}{2}\left[\left(\omega_{p e}^{2}+\omega_{\mathrm{LO}}^{2}\right)^{2}-4 \omega_{p e}^{2} \omega_{\mathrm{TO}}^{2}\right]^{1 / 2}
$$

where $\omega_{\mathrm{TO}}=\omega_{k}$ is the TO-mode frequency at $k=0, \omega_{\mathrm{LO}}^{2}=\omega_{\mathrm{TO}}^{2}+\omega_{p i}^{2}$, and $\omega_{p i}^{2}=4 \pi N Z^{2} / \varepsilon_{\infty} M^{\prime}$. These frequencies (related to the $\omega_{\mathrm{TO}}$ ) are shown in Fig. 1 as functions of the electron concentration, namely, $\omega_{p e} / \omega_{\mathrm{TO}}$. The upper line begins at $\omega_{\mathrm{LO}}$ and tends to the electron plasma frequency $\omega_{p e}$. The lower frequency starts as $\omega_{p e} \omega_{\mathrm{TO}} / \omega_{\mathrm{LO}}$ and then approaches $\omega_{\mathrm{TO}}$. In other words, observing in the optic range the longitudinal phonon mode and adding electrons, we see a transition of the longitudinal phonon frequency from $\omega_{\mathrm{LO}}$ to $\omega_{\mathrm{TO}}$. This is a result of the Coulomb screening.

We can compare Eq. (34) with the theory of Hon and Faust [11. Since in that theory the electron-phonon interaction $\zeta_{\mathrm{LO}}(\mathbf{p})$ as well as the electronic scattering $\gamma(\mathbf{p})$ were ignored, the phonon frequency and the deformation-optic constant were not renormalized. Then Eq. (34) can be rewritten as

$$
\chi(0, \omega)=\frac{\left(4 \pi g_{E}\right)^{2}}{\varepsilon_{\infty} \varepsilon(0, \omega)}\left[\varepsilon_{e}(0, \omega) A^{2} \chi_{I} / \varepsilon_{\infty}-\varepsilon_{\infty} / 4 \pi-2 A \chi_{I}\right],
$$

where $\chi_{I}=N Z^{2} / M^{\prime}\left(\omega_{\text {TO }}^{2}-i \omega \Gamma^{\text {nat }}-\omega^{2}\right), A=C \omega_{\text {TO }}^{2} M^{\prime} \varepsilon_{\infty} / 4 \pi N Z^{2}$, and $C=$ $g_{\mathrm{LO}} Z / g_{E} M \omega_{\text {TO }}^{2}$ is the Faust-Henry coefficient. Now we see, that the expression (37) coincides with the result of Hon and Faust [see, e.g. the paper [13], Eq. (3.1)].

For $k \neq 0$, Eq. (23) includes the dielectric function (19) which differs from the Lindhard-Mermin expression. Second, the condition determining frequencies and damping of the phonon-plasmon coupled modes

$$
\left(\tilde{\omega}_{j}^{2}-i \omega \Gamma^{\text {nat }}-\omega^{2}\right) \varepsilon_{e}(\mathbf{k}, \omega)+4 \pi N \widetilde{Z} \bar{Z} / M^{\prime}=0
$$

contains the phonon frequency $\tilde{\omega}$ and the ionic charge renormalized by the electronphonon interaction $\zeta_{\mathrm{LO}}(\mathbf{p})$. Third, the electro-optic coupling $g_{E}$ (21) is modified due to the light scattering $\gamma(\mathbf{p})$ from the electron-hole pairs. This effect is not canceled in the product $\tilde{g}_{E} \bar{g}_{E}$ of Eq. (23) even in the absence of the electron-phonon interaction $\zeta_{\mathrm{LO}}(\mathbf{p})$. The expansion of $\tilde{g}_{E}$ has the form

$$
\tilde{g}_{E}=g_{E}-\frac{i e \nu_{0} k\left\langle v_{z}^{2} \gamma(\mathbf{p})\right\rangle}{\left(\omega+i \tau^{-1}\right)^{2}}
$$

for $|\kappa| \ll 1$ and

$$
\tilde{g}_{E}=g_{E}+e \nu_{0} k^{-2}\left(\omega+i \tau^{-1}\right)\langle\gamma(\mathbf{p})(-\pi / 2-i \omega / k v) \delta(\mu) / v\rangle
$$


for $|\kappa| \gg 1$. We note, that the term $\tilde{g}_{E} \bar{g}_{E}$ has the largest imaginary part for $\omega \tau \simeq 1$ and then results more in the line-shape asymmetry.

Schematicly, the dispersion of phonon-plasmon modes is shown in Fig. 2. There are two main specularity of this figure. First, the behavior of the upper mode near the line $\omega=k v_{F}$. Around this line $\left(\tau^{-1}<\omega-k v_{F}<<k v_{F}\right)$ the dielectric function (19) has a singularity

$$
\varepsilon_{e}(k, \omega)=\varepsilon_{\infty}+\varepsilon_{\infty} \frac{k_{0}^{2}}{k^{2}}\left\{1-\frac{\omega}{2 k v_{F}}\left[\frac{1}{2} \ln \frac{4 k^{2} v_{F}^{2}}{\left(\omega-k v_{F}\right)^{2}+\tau^{-2}}-i \frac{\tau^{-1}}{\omega-k v_{F}}\right]\right\} .
$$

Because of this singularity, the upper mode approaches the asymptote $\omega=k v_{F}$, while the wave vector $k$ increases. Second, in the region $k v_{F} \gg \omega$, there is always one mode which mainly has phonon character. The reason of that is the decrease with $k$ of the imaginary part of the dielectric function (26).

\section{Discussion}

Now we consider obtained results in a simplest way. Let us assume that the electronic scattering is negligibly small, $\gamma(\mathbf{p})=0$. The second term in the parentheses of Eq. (14) is less than the first one in both limiting cases $\kappa \ll 1$ and $\kappa \gg 1$. We neglect this term at all. We also do not take into account the ionic charge renormalization because it vanishes at small values of $\kappa$. Then we can use the expression (25) not only for the dielectric function $\varepsilon_{e}(\mathbf{k}, \omega)$, Eq. (19), but also for the renormalized phonon frequency $\tilde{\omega}$, Eq. (14).

Results of the numerical calculations of the Raman spectra, Eq. (23), in this approximation are shown in Figs. 3 and 4 for two values of the electron-phonon coupling $\lambda_{e p h}=\nu_{0}\left\langle\zeta^{2}(\mathbf{p})\right\rangle / \omega_{0}^{3} M^{\prime} N \simeq p_{F} a m^{*} / m$. We take values of the Faust-Henry coefficient $C=-0.5$ and the phonon natural width $\Gamma^{\text {nat }}=10^{-2} \omega_{\mathrm{TO}}$. The electron collision rate is taken as $\tau^{-1}=10^{-1} \omega_{\mathrm{TO}}$, which is the usual value for heavily doped semiconductors [13, 19]. The wave vector $k$ and the Thomas-Fermi parameter $k_{0}$ are given in units of $\omega_{\mathrm{TO}} / v_{F}$, and the frequency $\omega$ in units of $\omega_{\mathrm{TO}}$ in all figures. Both these figures correspond to the case of small carrier numbers $\omega_{p e}<\omega_{\mathrm{TO}}$ (see, the right panel of Fig. 2; for the quadratic electron spectrum $\omega_{p e}=k_{0} v_{F} / \sqrt{3}$ ). The left peak mainly has a plasmon character and the right peak is mainly the LO phonon. We put the ion plasmon frequency $\omega_{p i}=\omega_{\mathrm{TO}}$, therefore $\omega_{\mathrm{LO}}=\sqrt{2} \omega_{\mathrm{TO}}$. When the wave vector $k$ approaches the boundary of the Landau damping region $k v_{F}>\omega$, the plasmon peak becomes broader and almost disappears at $k=0.8$. The broad continuum in the region $k v_{F}>\omega$ results from the excitation of electron-hole pairs. The intensity of the plasmon peak becomes larger in the comparison to the phonon peak, when the electron-phonon interaction $\lambda_{e p h}$ increases.

The $k$-dispersion of the plasmon (the peak position of the Raman spectra as a function of $k$ ), the line width (the full width at half maximum), and the line 
asymmetry (the frequency difference between the right and left wings of the resonance line at half maximum) are shown in Figs. $5-6$, all in units of $\omega_{\text {rmTO }}$. The width and asymmetry become much larger while the plasmon peak is immersed in the electron-hole continuum. The maximum in this region of spectra is nothing but the electron-hole contribution mainly. In Fig. 6, we see for $\lambda_{e p h}=0.1$ how close this maximum is located to the line $\omega=k v_{F}$.

The behavior of the phonon peak around $\omega=\omega_{\mathrm{LO}}$ with increasing of $k$ is shown in Fig. 7. While the wave vector increases from $k=0$ to $k=1.7$, the phonon peak is evidently shifted to the higher frequency and becomes broader. This is effect of the Landau damping (see Fig. 2, the right panel). But then, $k>1.75$, this peak appears at the lower frequency, $\omega \simeq 1.4$ and becomes more sharp for $k>2.2$, because the Landau damping decreases with $k$ [see Eq. (26) $]$.

The Raman spectra for heavily doped semiconductors and metals are shown in Figs. 8 - 9 (see also the left panel of Fig. 2). The phonon peak is located now around $\omega \sim \omega_{\mathrm{TO}}$ instead of $\omega \sim \omega_{\mathrm{LO}}$. This is effect of the Coulomb screening: carriers decrease the frequency of the LO mode from $\omega_{\mathrm{LO}}$ to $\omega_{\mathrm{TO}}$. We see also that the el-ph interaction suppresses the phonon peak.

The effect of the Coulomb screening and the el-ph interaction on the phonon mode is clearly seen in Figs. 10 - 11, where the phonon part of spectra is shown in detail. The lines are very asymmetric. The phonon dispersion, the line width, and the line asymmetry as $k$-functions are shown in Fig. 12 . We see a singularity at $k \simeq \omega / v_{F}$. It is interesting to estimate the value of the phonon dispersion. With the help of Fig. 11, we wind $d \omega / d k \leq 10^{-1} v_{F}$. From the other side, using Eqs. (26), (29), we find for the phonon dispersion $\omega^{2}=\omega_{\text {TO }}^{2}+\omega_{p i}^{2} k^{2} / k_{0}^{2}$, which well corresponds with the previous estimation for our values of $k_{0}$ and $\omega_{p i}$. Note, that these estimations confirm

the adiabatic approximation, since the value of dispersion $s=\omega_{p i} / k_{0} \sim v_{F} \sqrt{m / M}$ contains the adiabatic parameter.

In Fig. 13, the dispersion, the line width, and the line asymmetry are shown for the plasmon peak in heavily doped semiconductors. Here the effect of the elph interaction on the phonon dispersion is weak, no influence on the width and asymmetry of the line is seen.

\section{Conclusions}

In conclusions, we emphasize first, that the result of the paper (23) describes the renormalization of the phonon frequencies, the effective ionic charge and the coupling constants due to the electron-phonon deformation interaction $\zeta_{j}(\mathbf{p})$. Second, it involves the $k$-dependent semiclassical dielectric function instead of the LindhardMermin expression. At last, the light scattering $\gamma(\mathbf{p})$ with excitations of the electronhole pairs gives not only an additional contribution $\chi_{e}$ in Eq. (23), but also modifies the electro-optic $g_{E}$ and deformation-optic $g_{j}$ coupling constants which become de- 
pendent on the frequency and momentum transfers.

The author acknowledges the kind hospitality of the Max-Plank-Institut für

Physik komplexer Systeme, Dresden, were this work was completed. The work was partially supported by the RFBR (project 01-02-16211).

\section{References}

[1] A. B. Migdal, Zh. Eksp. Teor. Fiz. 34, 1438 (1958) [Sov. Phys. JETP 7, 996 (1958)].

[2] A. A. Abrikosov, L. P. Gor'kov, I. Ye. Dzyaloshinskii, Methods of Quantum Field Theory in Statistical Physics, (Prentice-Hall, Englewood Cliffs, NJ, 1963).

[3] A. S. Alexandrov and J. R. Schrieffer, Phys. Rev. B 56, 13731 (1997).

[4] M. Born and Kung Huang, Dynamical Theory of Crystal Lattices (Oxford University Press, New York, 1954).

[5] E. G. Brovman and Yu. Kagan, Zh. Eksp. Teor. Fiz. 52, 557 (1967) [Sov. Phys. JETP 25, 365 (1967)].

[6] V. M. Kontorovich, Usp. Fiz. Nauk 142, 265 (1984) [Sov. Phys. Uspekhi 27, $134(1984)]$.

[7] M. Reizer, Phys. Rev. B 61, 40 (2000).

[8] V. L. Gurevich, A. I. Larkin, and Yu. A. Firsov, Fiz. Tv. Tela 4, 185 (1962).

[9] L. A. Falkovsky, Phys. Rev. B 66, 020302-1 (2002); L. A. Falkovsky, Zh. Eksp. Teor. Fiz. 122, 411 (2002) [Sov. Phys. JETP 95, No 2(8) (2002)].

[10] G. Abstreiter, M. Cardona, and A. Pinczuk, Light Scattering in Solids, Topics in Applied Physics, vol. 54 edited by M. Cardona and G. Güntherodt (Springer, Berlin, 1984), p. 5.

[11] D.T. Hon and W. L. Faust, Appl. Phys. 1, 241 (1973).

[12] B. H. Bairamov, I. P. Ipatova, V. A. Milorava, V. V. Toporov, K. Naukkarinen, T. Tuomi, G. Irmer, and J. Monecke, Phys. Rev. B 38, 5722 (1988).

[13] L. Artús, R. Cuscó, and J. Ibánez, Phys. Rev. B 60, 5456 (1999).

[14] N. D. Mermin, Phys. Rev. B 1, 2362 (1970).

[15] L. A. Falkovsky and E. G. Mishchenko, Phys. Rev. B (1995). 
[16] A. A. Abrikosov and V. M. Genkin, Zh. Eksp. Teor. Fiz. 65 , 842 (1974); [Sov. Phys. JETP 38, 417 (1974)].

[17] A. A. Abrikosov and L. A. Falkovsky, Zh. Eksp. Teor. Fiz. 40 , 262 (1961); [Sov. Phys. JETP 13, 179 (1961)].

[18] A. Zawadowski and M. Cardona, Phys. Rev. B 42, 10732 (1990).

[19] R. Fukasawa and S. Perkowitz, Phys. Rev. B 50, 14119 (1994). 


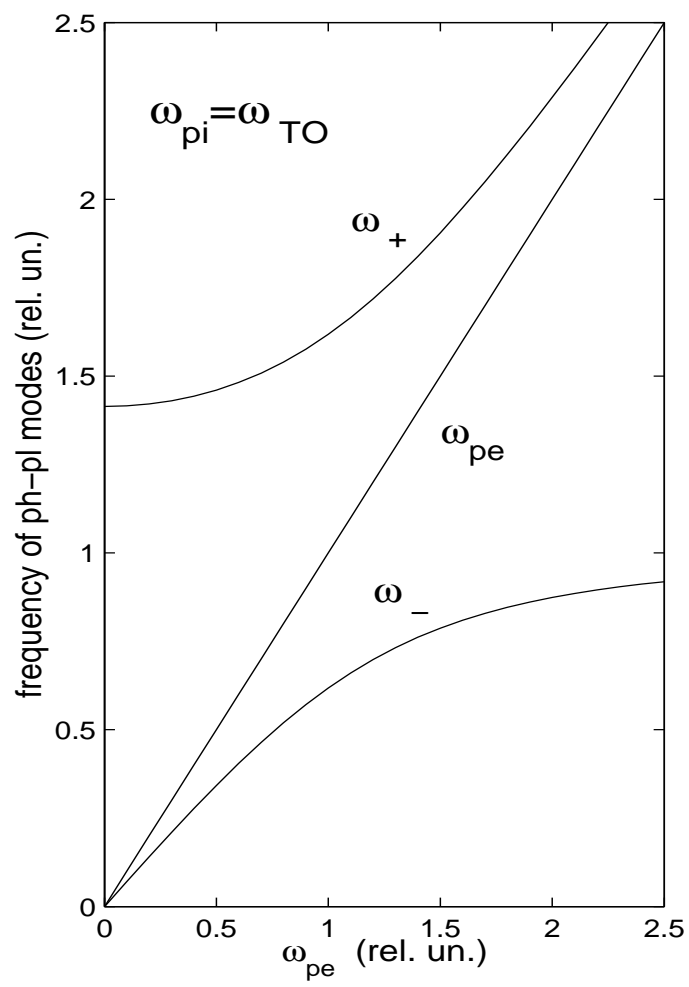

Figure 1: Frequencies (in units of $\omega_{\mathrm{TO}}$ ) of the phonon-plasmon modes at $k=0$ in dependence of the free carrier concentration, namely, of the electron plasma frequency (in units of $\omega_{\mathrm{TO}}$ ). We set the ion plasma frequency $\omega_{p i}=\omega_{\mathrm{TO}}$ in the absence of the free carriers. Then $\omega_{\mathrm{LO}} / \omega_{\mathrm{TO}}=\sqrt{2}$. 


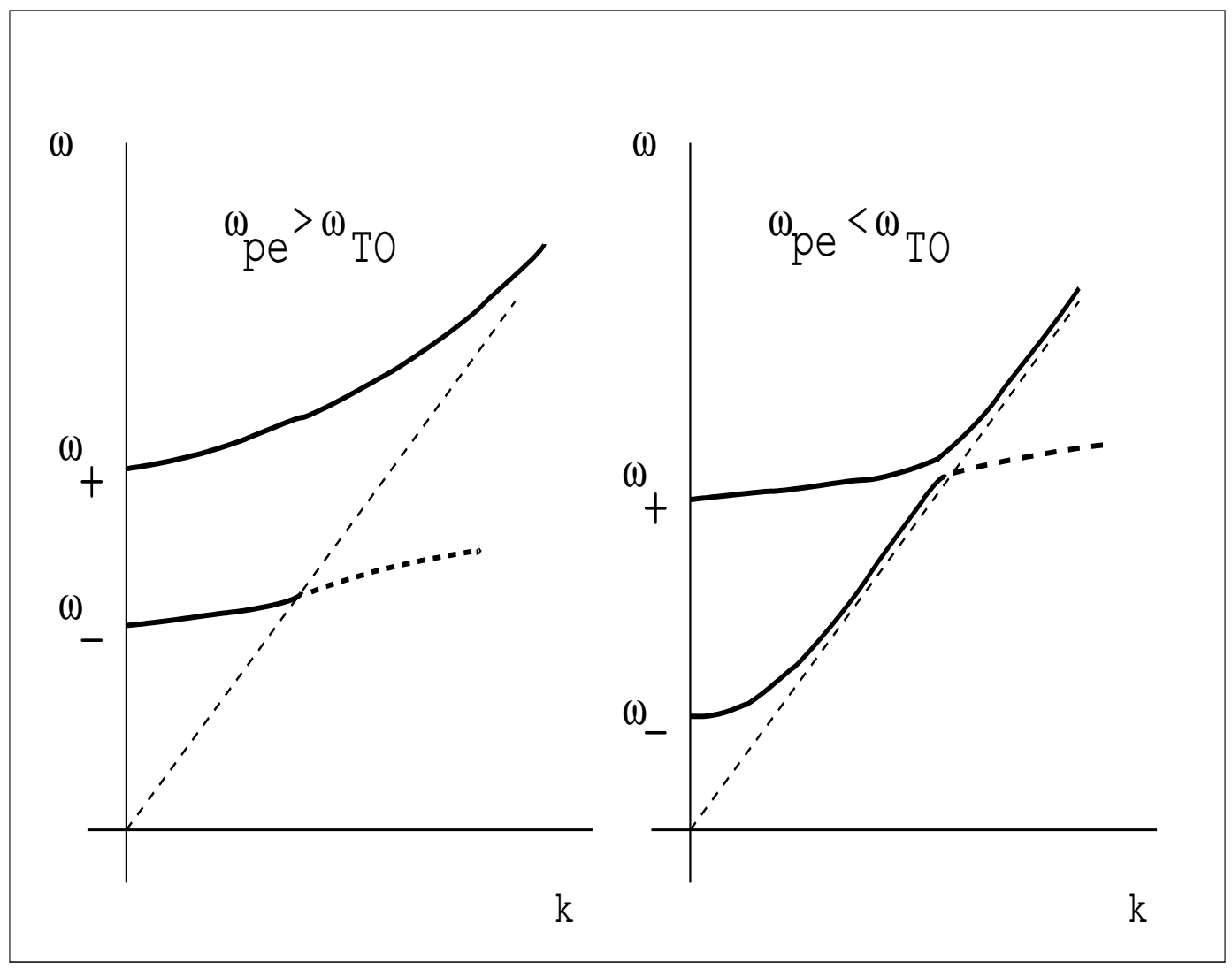

Figure 2: Schematic drawing of the dispersion of phonon-plasmon modes for the metallic $\left(\omega_{p e}>\omega_{\mathrm{TO}}\right.$, left panel) and semiconducting $\left(\omega_{p e}<\omega_{\mathrm{TO}}\right.$, right panel) carrier concentration. The dashed straight lines separate the domain $k v_{F}>\omega$, where the Landau damping exists; the dashed curves represent damped modes there. 


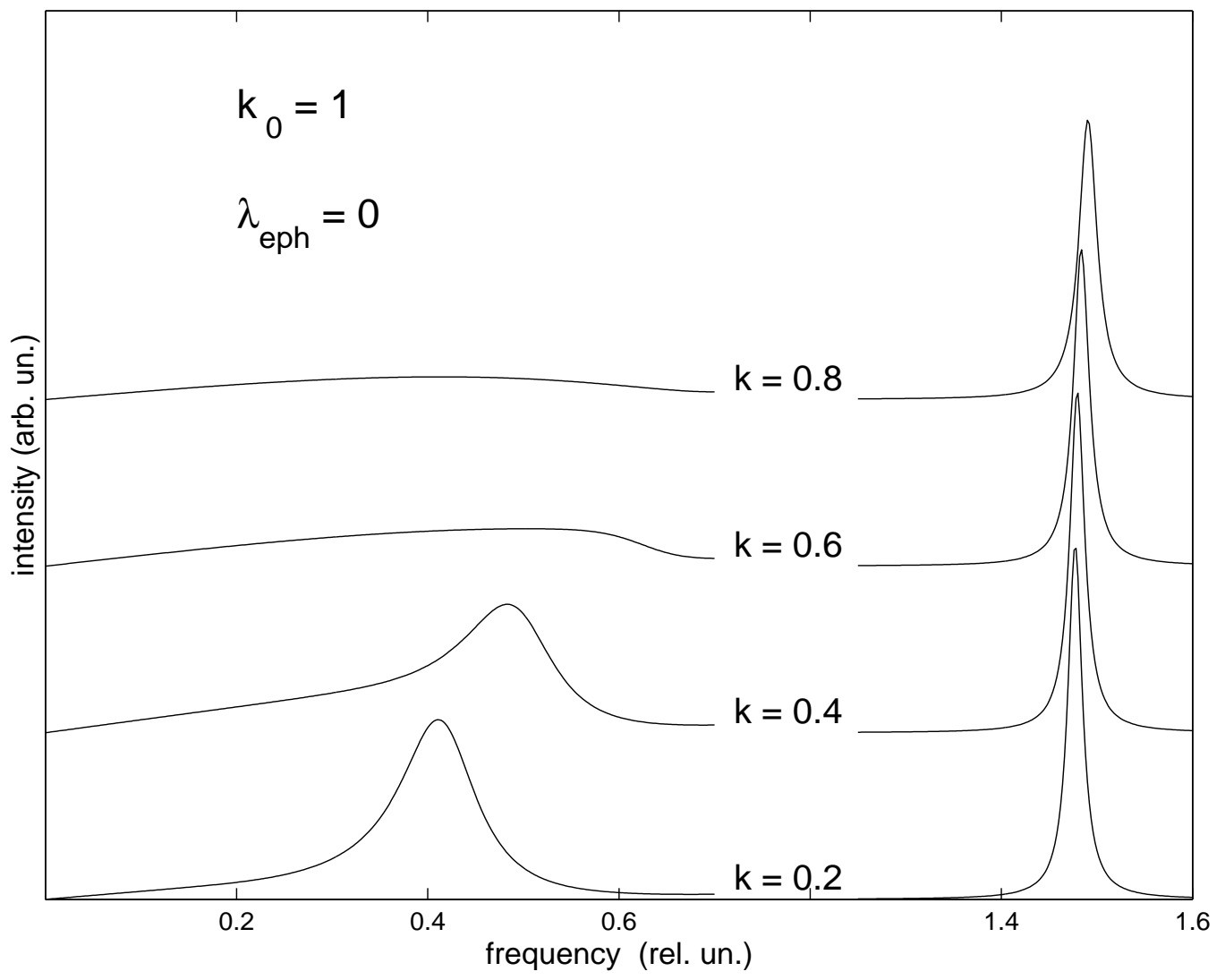

Figure 3: Raman spectra from a semiconductor with low carrier concentration as a function of the frequency transfer $\omega$ for denoted values of the momentum transfer $k$, the Thomas-Fermi parameter $k_{0}$ (in units of $\omega_{\mathrm{TO}} / v_{F}$ ), and the electron-phonon coupling constant $\lambda_{e p h}$. We set the ion plasma frequency $\omega_{p i}=\omega_{\mathrm{TO}}$, the phonon natural width $\Gamma^{\text {nat }} / \omega_{\mathrm{TO}}=10^{-2}$, and the carrier relaxation rate $\tau^{-1} / \Gamma^{\text {nat }}=10$. 




Figure 4: Same as Fig. 3 for $\lambda_{e p h}=0.1$. 




Figure 5: The plasmon dispersion as a function of $k$ in units of $\omega_{\mathrm{TO}} / v_{F}$ (the position in units of $\omega_{\text {TO }}$ of the line peak of Raman spectra; upper part of the figure, solid line). In the bottom, the line width (the full width at half maximum, dashed line), and the line asymmetry (the difference between the right and left wings at half maximum, dash-dotted line) in units of $\omega_{\mathrm{TO}}$. The Landau damping exists to the right of the dotted line $\omega=k v_{F}$. 


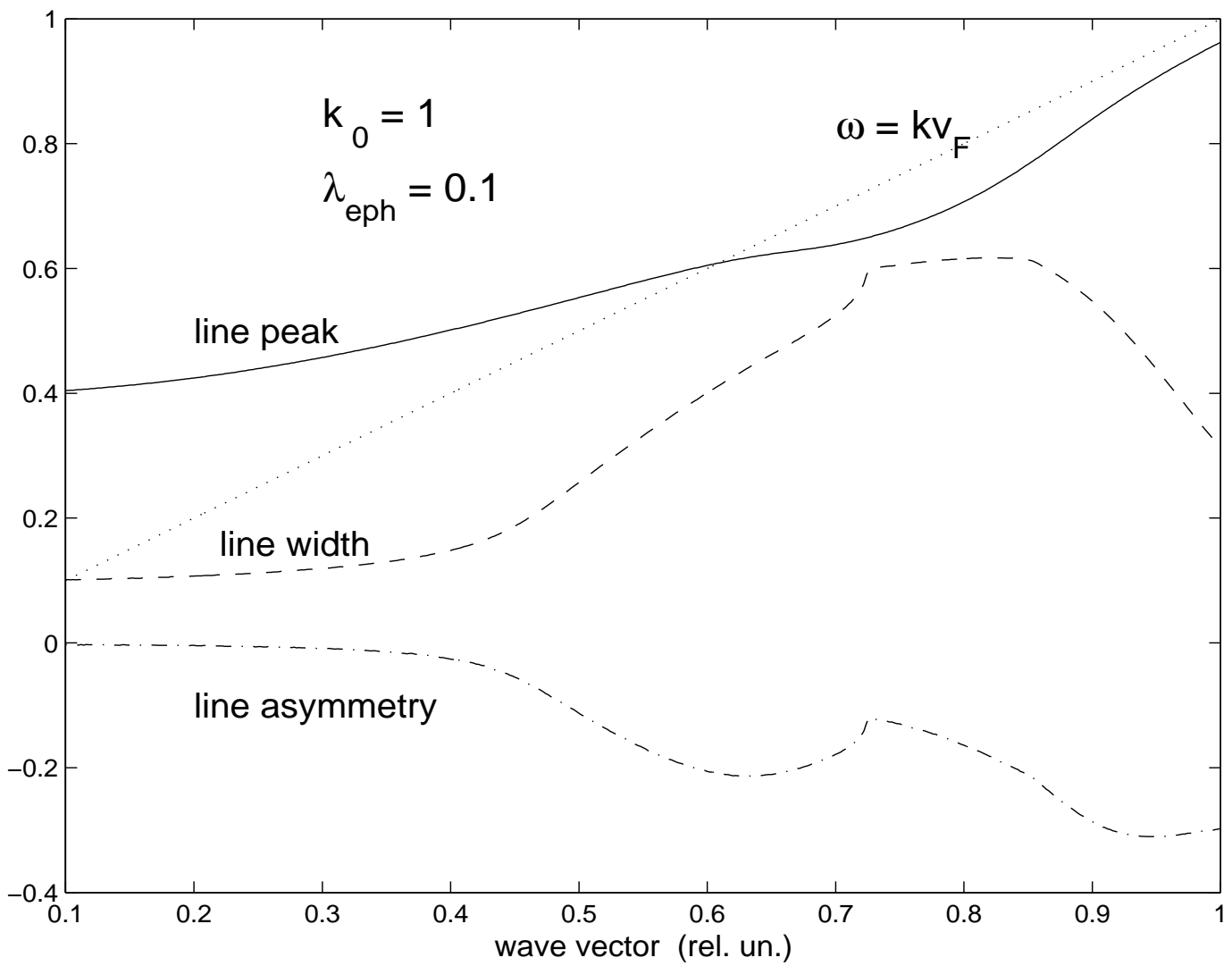

Figure 6: Same as Fig. 5 for the electron-phonon coupling $\lambda_{\text {eph }}=0.1$. 


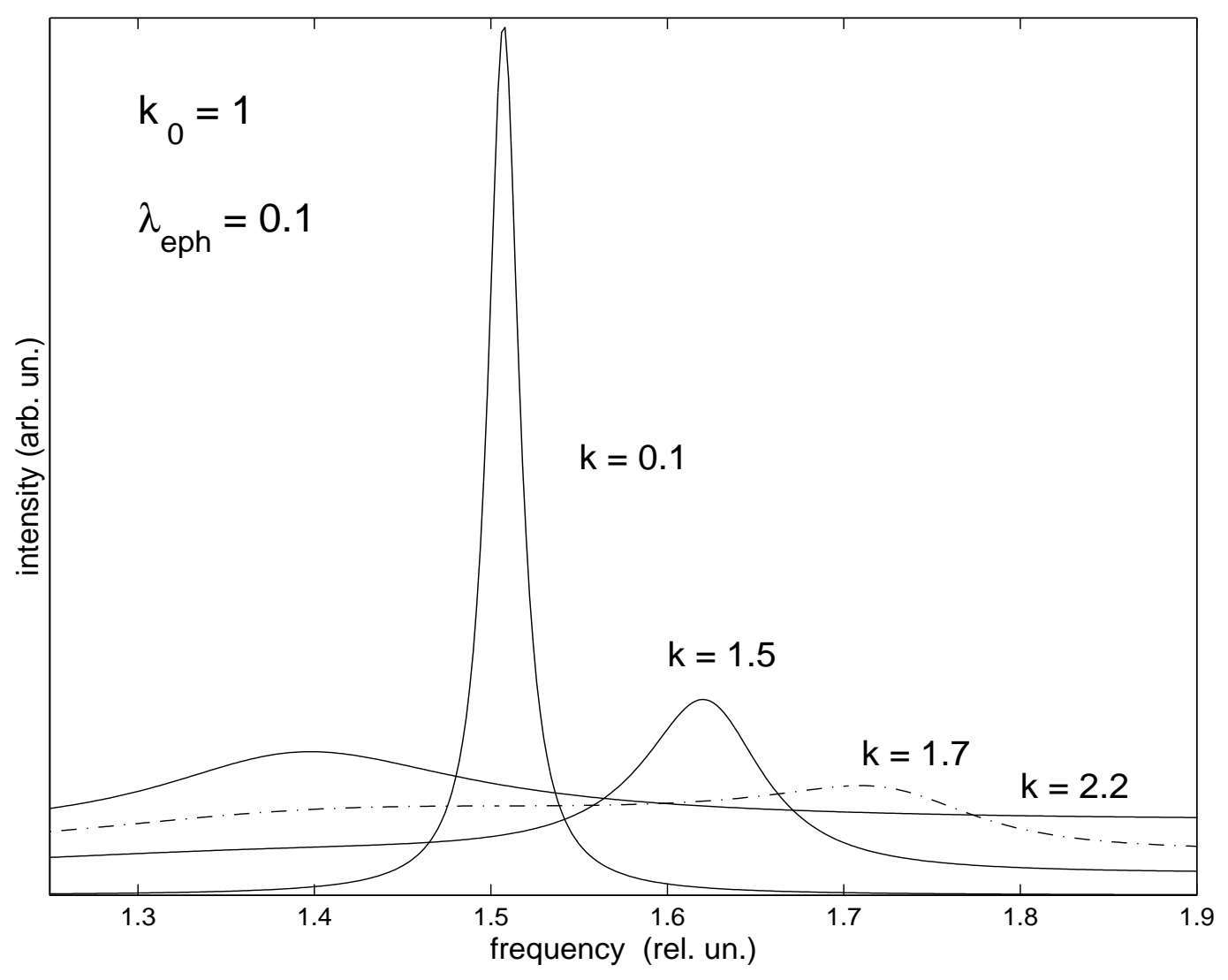

Figure 7: The LO phonon Raman spectra for large momentum transfers $k$. 


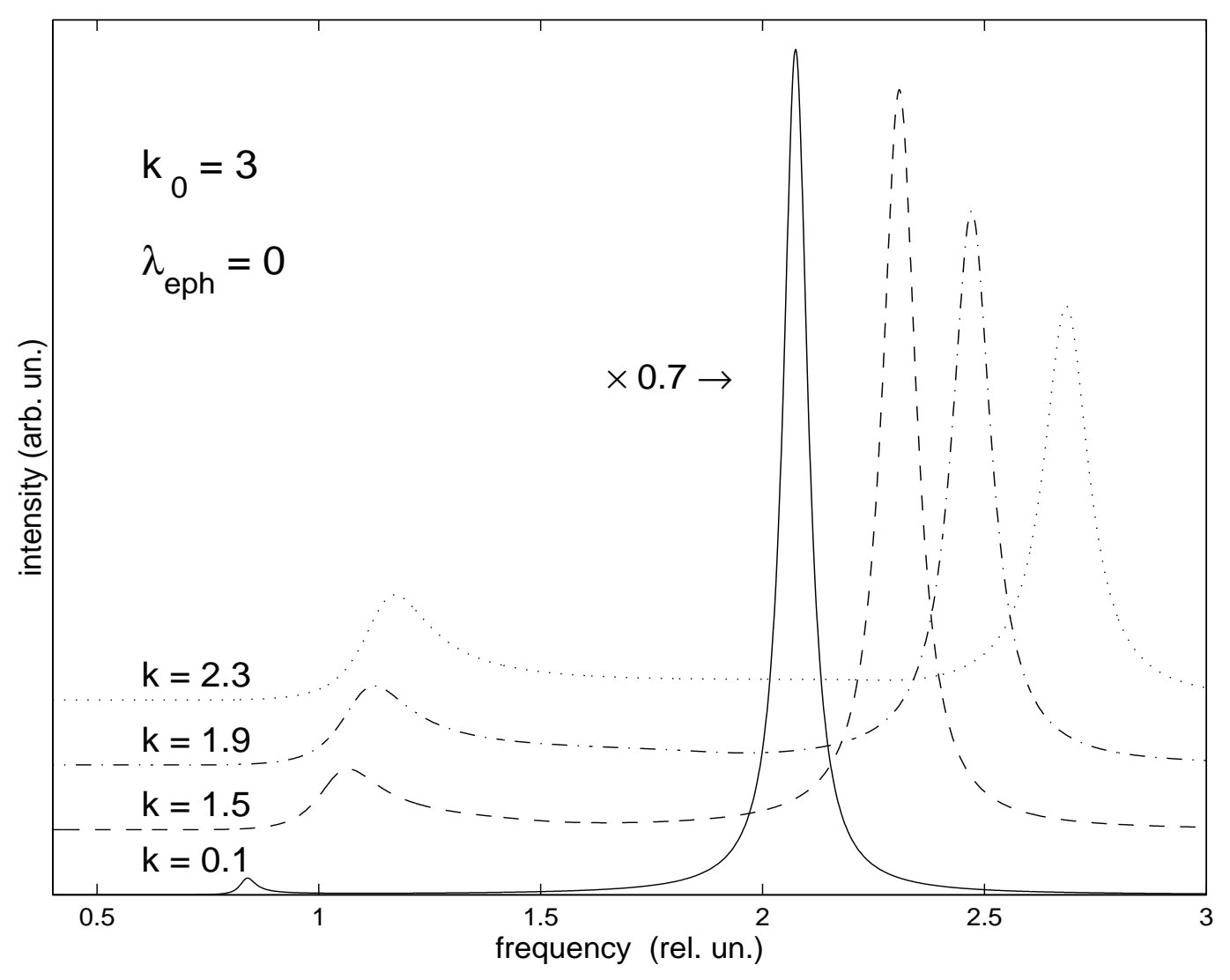

Figure 8: Raman spectra from a heavily doped semiconductor; notations as in Fig. 1. 




Figure 9: Same as in Fig. 8 for $\lambda_{e p h}=0.2$. 


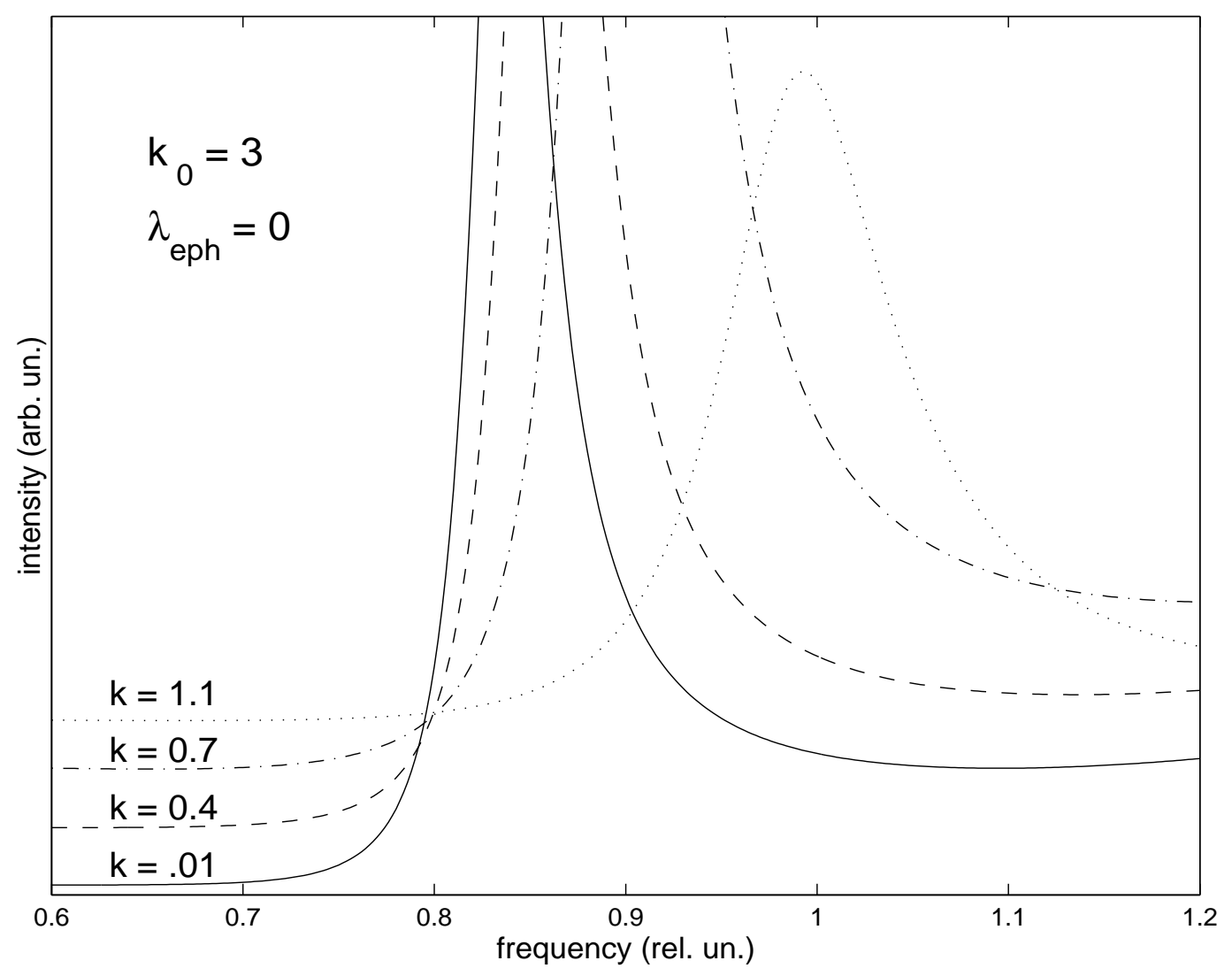

Figure 10: The LO phonon part of the Raman spectra from heavily doped semiconductor for various momentum transfers. 


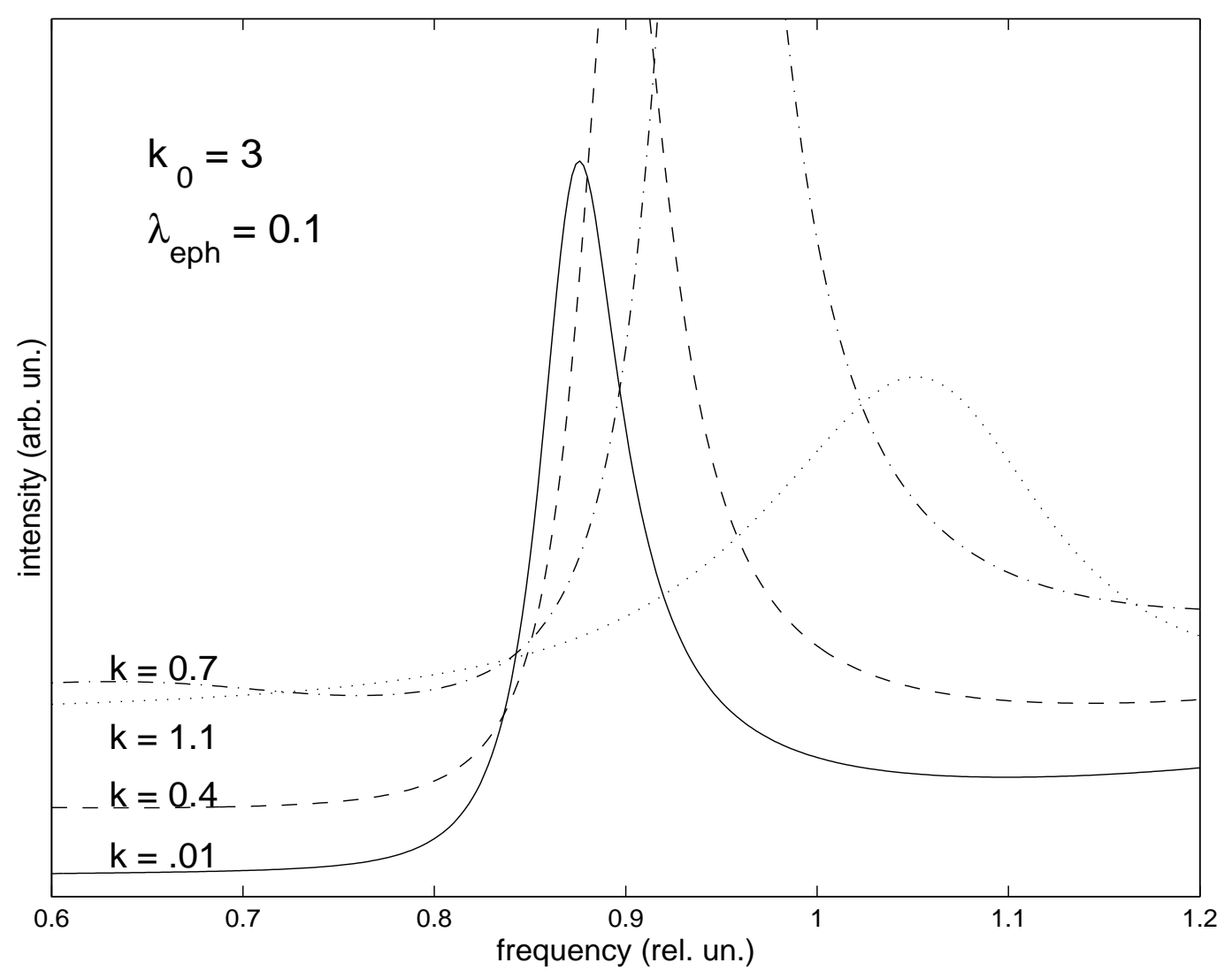

Figure 11: Same as in Fig 10, but the el-ph interaction is taken into account. 


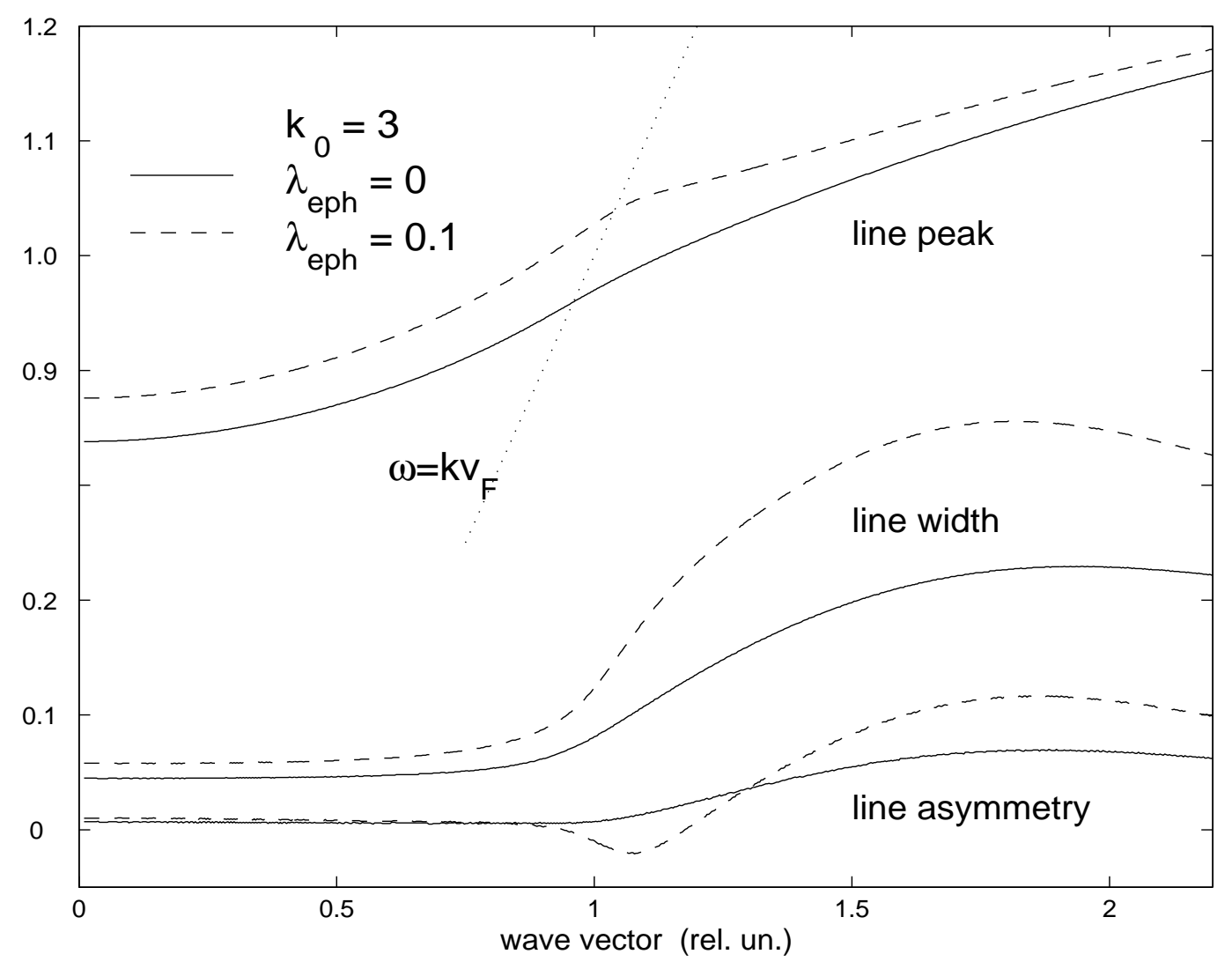

Figure 12: Dispersion of the phonon peak (upper), the line width and the line asymmetry (bottom). The boundary of the Landau damping region is shown as a dotted line. 


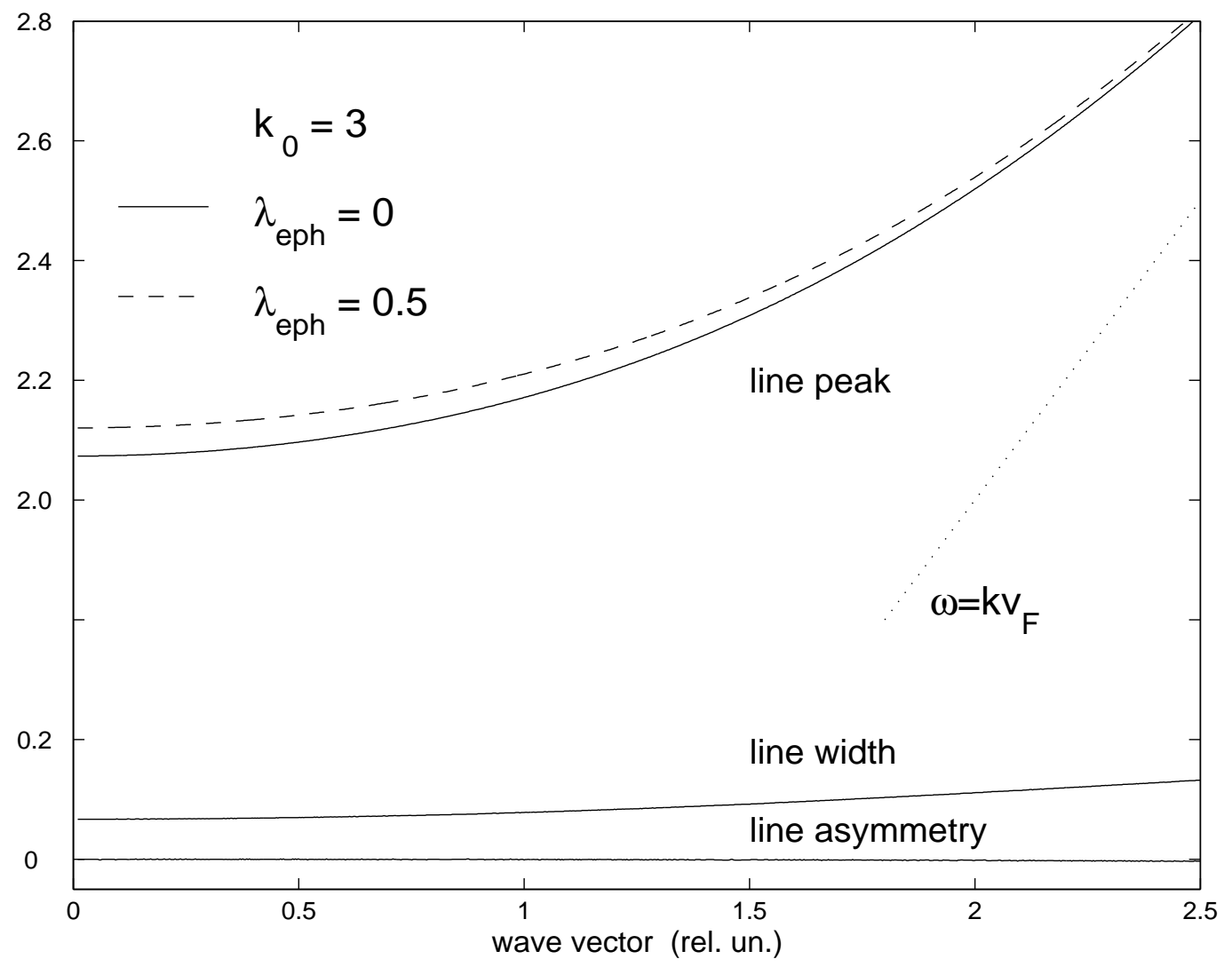

Figure 13: Dispersion of the plasmon peak. 